\title{
Cristianesimo e cultura classica. Modalità retoriche in alcuni testi cristiani (I ex.-II in.)
}

\author{
Emanuela PRINZIVALLI \\ Università di Roma «La Sapienza» \\ emanuela.prinzivalli@uniroma1.it
}

A distanza di più di venti anni dalla loro pubblicazione sono ancora pienamente valide le seguenti riflessioni di Averil Cameron ${ }^{1}$ :

Like the good Jewish teacher he was, Jesus taught in words, especially in parable. Within the first decades after his death came that sophisticated instrument, Pauline rhetoric, and only after it came the Gospels-rewritings, or recreations, of the text of the life of Jesus. At no point that we can now recapture was there a first Christianity distinct from his verbal expression [...]. Early Christianity was not purely a matter of ritual or ethical behaviour, or of miracles cures done by a wonder-worker and his successors; it was always a matter of teaching, of interpretation, and of definition. As Christ was the Word, so Christianity was its discourse or discourses. It is the relation of those discourses to their wider cultural context in the Roman Empire that needs to be explored again.

Può sembrare un'ovvietà dire che il cristianesimo $f u$ sin dall'inizio i suoi discorsi: ma, se riflettiamo un attimo, non è così. I discorsi, che siano quelli delle lettere paoline, cioè degli scritti più antichi all'interno del NT, o siano quelli ricreati negli Atti degli Apostoli, sono realmente 1'unica cosa che abbiamo della primissima fase del movimento di Gesù, e la preminenza del discorso, nelle sue varie articolazioni e generi (si pensi all'omiletica), rimane una costante del cristianesimo. Le testimonianze iconografiche, quelle archeologiche, quelle epigrafiche, dunque il complesso delle altre fonti cristiane, vengono dopo, fra II e III secolo. Ci sono altresì giunte alcune notizie da parte degli storici, un fatto eccezionale per un gruppo religioso dalle origini così oscure $^{2}$ : la più antica, il cosiddetto Testimonium Flavianum del giudeo Flavio Giu-

${ }^{1}$ A. Cameron, Christianity and the Rhetoric of Empire. The Development of the Christian Discourse, Berkeley etc., 1991, pp. 31-32.

${ }^{2} \mathrm{E}$ ' necessario ricordare che le fonti non cristiane su Gesù non sono affatto trascurabili, in paragone a quanto avviene abitualmente per eventi prodottisi nella periferia dell'Impero romano. Periodicamente, dall'Illuminismo in poi, risorge una posizione miticista, che rispolvera, per negare l'esistenza di Gesù, l'obiezione che nel primo secolo ci sono solo fonti di adepti a parlare di lui. Questa è una distorsione di prospettiva, dato che gli storici romani non si preoccupano di segnalare neppure i fatti importanti avvenuti in 
seppe, nonostante qualche ampliamento di mano cristiana, appare fondamentalmente autentica ${ }^{3}$. Eppure è molto poco. Quello che fin dall'inizio campeggia, si spande, prolifica è la formidabile capacità di comunicazione dei cristiani, o almeno di alcuni cristiani, che sono stati capaci di lasciarci un ampio corpo di scritti fin dalle prime origini del nuovo fenomeno. Sono scritti in lingua greca. E questa è la prima cosa che conta, il primo passo della conquista lenta e silenziosa di adepti da parte del messaggio evangelico nei primi tre secoli. Cercherò di ripercorrere alcune tappe di questo processo, partendo appunto dal primo passo: l'uso della lingua greca.

\section{IL PRIMO PASSO: L'USO DELLA LINGUA GRECA}

L'impero romano eredita l'universalismo ellenistico, conseguente alle conquiste di Alessandro Magno, mutuandone anche la visione culturale - per cui possiamo parlare di civiltà greco-romana-, nonché i principi dell'istruzione: in quest'ambito la retorica, come è noto, si identifica con la possibilità stessa di esprimersi, di farsi capire e di farsi ascoltare. Naturalmente, il punto di partenza imprescindibile è che si sia in grado di usare le lingue universali dell'impero, il latino, la lingua dell'amministrazione, e il greco, la lingua della cultura, oltre che degli scambi commerciali nel bacino mediterraneo.

La formula latina che più si avvicina al concetto moderno di bilinguismo, cioè utraque lingua, non indica per i romani né giustapposizione né opposizione di partenza di due lingue e culture, piuttosto un rapporto di unità e complementarità fra di esse, essendo il latino e il greco due facce indissolubilmente interrelate di un'identità dinamica, dove il greco viene incluso nella cultura romana in opposizione al mondo barbaro. Stante questa mentalità di fondo, nella visione greco-romana le sole lingue nel senso proprio del termine sono il greco e il latino ${ }^{4}$. La traduzione in queste lingue è dunque un passaggio necessario, per chi voglia rendersi visibile in quanto udibile e ascoltabile nell'impero greco-romano.

Giudea sotto Ponzio Pilato, che conosciamo da fonti giudaiche, e solo un'iscrizione frammentaria ritrovata a Caesarea Maritima ci dice che fu prefetto di Giudea. Paradossalmente il solo storico romano che lo menziona è Tacito, all'inizio del II secolo, ma nell'ambito della notizia riguardante Gesù. Sulla fallacia delle argomentazioni dei miticisti cfr. B. D. Ehrmann, Did Jesus exist? The Historical Argument for Jesus of Nazareth, New York, 2012.

${ }^{3}$ Per una ricognizione accurata delle diverse posizioni della critica: S. Bardet, Le Testimonium Flavianum. Examen historique, considérations historiographiques, Paris, 2002. Permangono posizioni di scetticismo totale: F. Parente, «Sulla doppia trasmissione, filologica ed ecclesiastica, del testo di Flavio Giuseppe: un contributo alla storia della ricezione della sua opera nel mondo cristiano», Rivista di Storia e Letteratura religiosa 36 (2000), pp. 9-25. P. Angelo Gramaglia, «Il Testimonium Flavianum. Analisi linguistica», Henoch 20 (1998), pp. 153-177, ma c'è anche chi sostiene l'autenticità integrale del testo, opportunamente interpretato: oltre allo stesso L. Bardet, op. cit., cfr. L. Troiani, «Il Gesù di Giuseppe Flavio», A. Pitta (ed.), «Il Gesù storico nelle fonti del I-II secolo». Atti del X Convegno di studi neotestamentari, Foligno, 11-13 settembre 2003, Ricerche storico bibliche 17.2 (2005), pp. 137-147; e Id., «Ancora sul cosiddetto Testimonium Flavianum» 2007 (www.christianismus.it).

${ }^{4}$ B. Rochette, Le latin dans le monde grec. Recherches sur la diffusion de la langue et des lettres latines dans les provinces hellénophones de l'Empire romain (Collection Latomus), Bruxelles 1997, p. 49. 
Se ci rivolgiamo a Paolo come personaggio rappresentativo delle origini cristiane o, comunque, come autore destinato a lasciare un'impronta indelebile nella successiva storia del cristianesimo ${ }^{5}$ (non si dimentichi che il NT è sotto il segno preponderante della sua influenza) immediatamente ci sovvengono le sue drastiche parole (1 Cor 1, 17-25) in merito al sovvertimento di valori che la fede in Gesù Cristo crocifisso comporta:

${ }^{17}$ Cristo infatti non mi ha mandato a battezzare, ma a predicare il vangelo; non però con sapienza di discorso, perché non venga svuotata la croce di Cristo. ${ }^{18} \mathrm{La}$ parola della croce infatti è stoltezza per quelli cha vanno in perdizione, ma per quelli che si salvano, per noi, è potenza di Dio. ${ }^{19}$ Sta scritto infatti: Distruggerò la sapienza dei sapienti e annullerò l'intelligenza degli intelligenti. ${ }^{20}$ Dov'è il sapiente? Dov'è il grammatico ( $(\rho \alpha \mu \mu \alpha \tau \varepsilon v ́ \varsigma)$ ? Dove mai il sottile ragionatore di questo mondo? Non ha forse Dio dimostrato stolta la sapienza di questo mondo? ${ }^{21}$ Poiché, infatti, per la sapienza di Dio il mondo, con tutta la sua sapienza, non ha conosciuto Dio, è piaciuto a Dio di salvare i credenti con la stoltezza della predicazione $(\delta i \grave{\alpha} \tau \tilde{\eta} \varsigma$

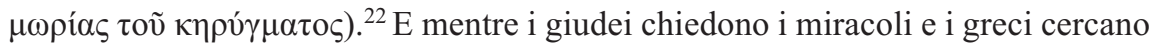
la sapienza, ${ }^{23}$ noi predichiamo Cristo crocifisso, scandalo per i giudei, stoltezza per i gentili; ${ }^{24} \mathrm{ma}$ per coloro che sono chiamati, sia giudei che greci, predichiamo Cristo potenza di Dio e sapienza di Dio. ${ }^{25}$ Perché ciò che è stoltezza di Dio è più sapiente degli uomini, e ciò che è debolezza di Dio è più forte degli uomini.

Le antitesi paoline non si limitano a opporre alla sapienza del mondo (v. 20: бopía

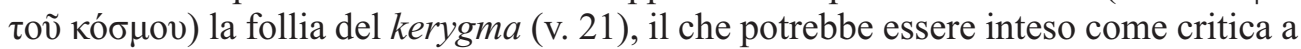
un modo di ragionare umano in senso generico, ma precisano in senso specifico l'opposizione al sistema di pensiero e di educazione greca. Sono eloquenti le sue

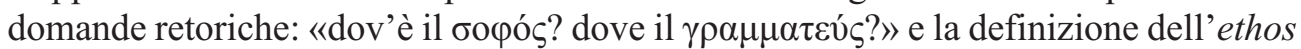
dei greci come quello di chi cerca la sapienza ("Е allora vuole dire non solo ricerca filosofica ma anche conoscenza dei meccanismi espressivi che permettono a una persona non solo di vivere, ma di farsi ascoltare, di inserirsi e di influenzare i poteri che governano la società, in forza dell'accostamento con il termine $\gamma \rho \alpha \mu \mu \alpha \tau \varepsilon v ́ \varsigma$, che nel greco post-classico assume il significato di chi ha a che fare con l'espressione linguistica del pensiero.

Tuttavia questo messaggio di radicale opposizione all' ethos mondano, o se si vuole di radicale rinnovamento dell'ethos, per la sua stessa natura di messaggio sal-

\footnotetext{
${ }^{5}$ Il Nuovo Testamento come canone, cioè come selezione di libri considerati sacri per i cristiani, attraverso un duplice dispositivo di inclusione e di esclusione, si forma, come è noto, nella seconda metà del II secolo: esso è sotto l'inequivocabile segno di Paolo. E' il suo pensiero infatti a marcare indelebilmente e a orientare la successiva esegesi degli scritti neotestamentari. Non solo le lettere paoline, deutero paoline e trito paoline, rappresentano il maggior numero di scritti inclusi nel NT, ma altre lettere risentono del suo influsso, come quelle attribuite a Pietro, il vangelo di Luca e gli Atti, da molti studiosi considerati un dittico inscindibile, hanno come scopo l'incontro e la conciliazione delle tendenze paoline e in generale ellenistiche con le varie tendenze della missione giudeocristiana: G. Aragione, E. Norelli (eds.), Le canon de Nouveau Testament. Regards nouveaux sur l'histoire de sa formation, Gèneve 2005; E. Thomassen (ed.), Canon and Canonicity. The Formation and Use of Scripture, Copenhagen 2010.
} 
vifico destinato, nell'ottica paolina, ad essere universale, deve essere persuasivo, convincente e quindi deve essere ri-detto secondo modalità che lo predispongano al successo. Laddove dunque il senso delle parole paoline starebbe a negare la possibilità di un confronto e di una interazione con il mondo della sapienza greca, la stessa urgenza del suo esprimersi, la foga asiana del suo procedere, i procedimenti retorici, destinati sì a un gruppo di fedeli in Cristo, che quindi sembrerebbero ormai fuori dai lacci di quella sapienza, ma che, come la lettera paolina implicitamente dimostra, ne sono continuamente sollecitati, rendono evidente la piattaforma culturale dalla quale Paolo non può prescindere e della quale è lui stesso partecipe, in quanto proveniente dal giudaismo ellenistico. L'interazione con il mondo, la cui sapienza viene negata, è intrinseca all'annuncio stesso di salvezza formulato da un oscuro galileo, che si esprimeva abitualmente in lingua aramaica, e si attua nel momento stesso della sua ritraduzione, ripetizione e riformulazione nella lingua greca, lingua madre di Paolo, e dei tanti missionari ellenisti come lui. Gesù stesso, come viene rappresentato nei Vangeli, possedeva una discreta conoscenza della lingua greca, avendo occasionalmente contatti con grecofoni ${ }^{6}$. In ogni caso, la diffusione in lingua greca del suo messaggio comincia praticamente durante la sua vita, in particolare in Gerusalemme, dove continuamente affluivano giudei grecofoni della diaspora e dove il greco era conosciuto a vari livelli.

Il messaggio evangelico, dunque, è subito presentato nella lingua dominante, il greco, di fatto diventata la prima lingua per i giudei della diaspora. L'incipiente formazione di un corpus di scritture cristiane non fa che riprodurre quella che era stata la vicenda delle scritture ebraiche nella diaspora, con la traduzione in greco, detta dei Settanta, fatta ad uso degli stessi giudei ormai grecofoni, ma accompagnata da tutta una serie di adattamenti alla mentalità greca e dalla composizione della Lettera di Aristea, che programmaticamente considera la sapienza giudaica come massima espressione di sapienza ellenica. Anzi la vicenda cristiana è più intensa e radicale in quanto non abbiamo traccia, stando a quello che a noi è giunto, di scritti cristiani delle origini in aramaico, e solo in alcuni casi abbiamo testimonianze indirette di una possibile composizione di vangeli in ebraico.

\section{LINGUA GRECA E RETORICA IN PAOLO E NEL PAOLO DI LUCA}

Se da Paolo autore di lettere passiamo per un attimo al Paolo rappresentato dal suo ammiratore Luca, il quale - non lo si dimentichi - compone gli Atti degli Apostoli secondo le regole di base della storiografia tucididea, mettendo in bocca ai vari personaggi discorsi che rappresentino efficacemente la posizione di ciascuno di essi, con-

\footnotetext{
${ }^{6}$ Si possono coglierne indizi nell'episodio della donna greca sirofenicia (Mc 7,25-30) e nel colloquio con Pilato (Mc 15, 2-5 e par.) e nella capacità di formulare giochi di parole mescolando aramaico e greco. Il prof.

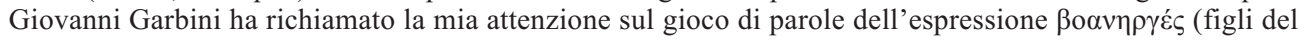
tuono) di Mc 3,17. In aramaico si sarebbe detto benerges, ma Gesù mescola con il verbo greco $\beta$ ó́ $\omega$.
} 
statiamo come nel discorso sull'Areopago, emblematico della evangelizzazione ai greci, la retorica di Paolo faccia uso di conoscenze filosofiche e persino di una citazione poetica dal poeta Arato. Il Paolo degli Atti, sulla scia del modo di procedere del proselitismo ebraico, cerca un aggancio nella cultura greca, un incontro a metà strada, privilegiando ciò che accomuna e giocando su una certa ambiguità ${ }^{7}$. Così l'altare «al Dio ignoto» trovato in Atene esprime secondo l'oratore Paolo la ricerca di un dio che possa fornire una risposta agli interrogativi degli uomini di tutte le nazioni, e che egli identifica con il dio creatore, provvidente e giudice, da lui annunciato. Nell' economia del discorso sull'Areopago, il tema messianico è solo accennato, viene completamente taciuta l'identificazione del proprio dio con il dio dei giudei e lo stesso monoteismo è presentato in modo sfumato e quindi condivisibile da chi avesse un'infarinatura filosofica. Di fatto Paolo esprime il suo messaggio in termini filosofici, una prospettiva che sarà praticata nel II secolo consapevolmente prima dai maestri cristiani gnostici e poi da quelli ortodossi. Il discorso paolino si arena sulla questione della resurrezione dei morti. A questo punto si apre un'altra problematica che qui debbo lasciare da parte, di carattere squisitamente filosofico e dottrinale, riguardante i capisaldi, in materia protologica, la creazione, ed escatologica, la resurrezione dei corpi, mutuati dal giudaismo, che consentirono al cristianesimo di non farsi riassorbire nelle filosofie ellenistiche ${ }^{8}$. Posso solo enunciarla perché debbo tenere la barra del timone diritta sul nostro tema, e quindi ritorno dal Paolo narrato da Luca al Paolo delle lettere autentiche. Capiamo perché Luca abbia potuto dare di Paolo una simile rappresentazione: quest'ultimo, lo abbiamo detto sopra, scrive secondo modalità retoriche. Una serie abbondante di studi contemporanei ha letto le sue lettere alla luce dei moduli della retorica deliberativa, anzi addirittura tenta di suddividerle secondo le partizioni di un discorso retorico, anche se questo tentativo non riesce a dare risultati univoci ${ }^{9}$. E' necessario a riguardo fare alcune precisazioni. L'interferenza tra epistolografia e retorica non può essere revocata in dubbio. Gli epistolari dell'antichità ci fanno capire quanta fosse la cura nella composizione di una lettera, a maggior ragione dispensata per il fatto che questa doveva sostituire il dialogo diretto con l'interlocutore e quindi raggiungere con la sola forza delle parole scritte quell'efficacia che non poteva essere ottenuta con altri mezzi. E nel caso di Paolo l'efficacia doveva di necessità essere massima in quanto le lettere corrispondono alla seconda, decisiva, fase della sua evangelizzazione ${ }^{10}$, allorché egli conferma, rafforza, guida da lontano le piccole comunità da lui fondate (la Lettera ai Romani, la più accurata anche dal punto di vista compositivo è un caso a parte, ancora più significativo dell'obbligo di essere efficace, in quanto si tratta di presentarsi a una comunità che lo conosce solo per sentito dire). La forma compositiva della lettera non ha però

\footnotetext{
${ }^{7}$ M. Sachot, La predicazione del Cristo. Genesi di una religione, Torino 1999 (ed. orig. 1998), 113s.

${ }^{8}$ Mi permetto di rinviare a: E. Prinzivalli, M. Simonetti, La teologia degli antichi cristiani (secoli $I-V$ ), Brescia 2012, pp. 209-294.

${ }^{9}$ Sui risultati contradditori $c f r$. R. Burnet, Épittres et lettres $I^{e}-I^{e}$ siècle. De Paul de Tarse à Polycarpe de Smyrne, Paris, 2003, p. 181.

${ }^{10}$ Cfr. M. Pesce, Le due fasi della predicazione di Paolo. Dall'evangelizzazione alla guida della comunità, Bologna, 1994.
} 
goduto nell'antichità di molte trattazioni teoriche specifiche sulla sua qualità letteraria, come è stato invece per i tre generi principali prosastici: l'oratoria, il dialogo filosofico e la storiografia. Kennedy, uno degli studiosi che più hanno esercitato un'influenza nel settore, sottolinea che gli antichi la inseriscono sulla scia imitativa di uno di questi generi, soprattutto dell'oratoria: «la parte centrale può assumere la forma di un discorso deliberativo, epidittico o giudiziario, con le partizioni tradizionali e tutte le peculiarità dell'inventio e dell'elocutio di un'orazione ${ }^{11}$. Tuttavia la gamma concreta delle situazioni e quindi dei modi di composizione di una lettera appare molto più complessa, basti considerare i ventuno typoi di lettere recensiti dallo Ps. Demetrio ${ }^{12}$ e i quarantuno recensiti dallo Ps. Libanio ${ }^{13}$. L'epistolografia, per quanto si accosti all'oratoria o la imiti, gode di libertà propria rispetto a questa, tanto più in quanto è un genere per sua natura multifunzionale e minore, arrivato solo gradualmente a interessare in modo specifico $i$ teorici antichi ${ }^{14}$. Esaminando quindi una lettera delle origini cristiane bisogna evitare di applicare ad essa in modo rigido lo schema standard di un'orazione. Gli studi più recenti di conseguenza sono sempre più avvertiti dell'opportunità di usare duttilità critica con l'epistolario paolino, tenendo presente la possibile compresenza di diversi typoi nell'ambito di una stessa lettera o la ripetizione dello schema base del discorso, evitando così classificazioni affrettate, basate sulle categorie del discorso, per definire una singola unità retorica ${ }^{15}$.

\section{MODALITÀ RETORICHE IN ALCUNI TESTI CRISTIANI FRA I E II SECOLO}

Amplio adesso il discorso da Paolo agli altri scritti neotestamentari, e con ciò debbo subito aprire una necessaria parentesi: parlando di NT avverto che sto facendo uso di una distinzione teologica inadeguata alla presente analisi che intende leggere i testi cristiani nel contesto culturale dei loro tempi e dal punto di vista storico letterario e che quindi, semmai, deve confrontare questi testi con la coeva produzione in lingua greca. Tuttavia alcuni studi sulla retorica dei testi cristiani assumono la categoria neotestamentaria come ambito di indagine ${ }^{16} \mathrm{e}$ in ogni caso un numero consistente di testi neotestamentari -ma non tutti!- sono anche i testi cristiani più antichi, perciò per comodità parlerò ora degli altri scritti neotestamentari. Ebbene, svariati studi sono stati

${ }^{11}$ G. A. Kennedy, Nuovo Testamento e critica retorica, Brescia, 2006 (ed. orig. Chapell Ill 1984), p. 110.

12 J. L. White, Light from Ancient Letters, Philadelphia, 1986, p. 189.

${ }^{13}$ J.T. Reed «The Epistle», J.E. Porter (ed.), Handbook of Classical Rhetoric in the Hellenistic Period 330. B.C.-A.D. 400, Leiden, 1997, p. 174 e 192. Reed scrive efficacemente: «i due generi (retorica ed epistolografia) possono essere stati fidanzati, ma sposati mai».

${ }^{14}$ J. T. Reed, op. cit., p. 191.

${ }^{15}$ Su tutto ciò cfr. J.-N. Aletti, Saint Paul. Épître aux Philippiens, Paris, 2005, pp. 24-25.

${ }^{16}$ Cito il caso del volume di V.K. Robbins, The Invention of Christian Discourse, I, Blandform Forum, Dorset 2009, che sviluppa l'analisi del «Rhetorolect», abbreviazione di «Rhetorical dialect» cristiano, cioè del particolare linguaggio formale o discorso che si può identificare a partire da temi peculiari, immagini, ragionamenti che formano un sistema di credenze e un modo di vivere. Robbins considera scorretta 
dedicati a definire le modalità e i termini della parenesi cristiana, giustamente considerata, in base alle risultanze delle fonti, un importante aspetto del pensiero e della pratica dell'antico cristianesimo. Se guardiamo a un volume miscellaneo abbastanza recente - interessante tanto nei suoi pregi quanto nei suoi difetti - Early Christian Paraenesis in Context, edito nel 2005, - nell'introduzione dei curatori James Starr e Troels Engberg-Pedersen troviamo, a partire dal primo problema, quello della definizione di parenesi, la consapevolezza dell'intreccio di influssi ma anche del ruolo preminente della tradizione greco-romana. Così infatti si esprimono i curatori: such questions (n.b. le questioni del concetto di parenesi, della sua fenomenologia, di quali testi possono essere inclusi in essa e se essa è un genere letterario) are relevant to the broader Graeco-Roman tradition, where the term itself has its origin, to the Jewish tradition, which partly overlaps with the Graeco-Roman tradition, and to the early Christian tradition, which completely overlaps with the other two (p. 2). Connesso con questa problematica è quella dello spartiacque fra giudaismo ed ellenismo, cioè se la parenesi sia un fenomeno greco-romano, o più generale, o se ci sia una versione più specificatamente giudaica e infine quale sia l'uso specifico cristiano. Tradotto a più ampio spettro, il problema, per quanto riguarda i primi scritti cristiani, è sempre quello di vedere come si esprimono i nuovi contenuti a partire da un tessuto linguistico culturale ellenistico e molto spesso, giudaico-ellenistico, considerato che non pochi di questi primi scritti appartengono ad autori di estrazione giudaica.

Un caso emblematico è costituito dalla Prima lettera di Clemente ai Corinzi (= IClem), che sorprende sia stata tralasciata dagli autori del volume miscellaneo sopra citato, in quanto potrebbe rientrare nella definizione di parenesi, essendo appunto una parenesi di particolare lunghezza. Come è noto, la comunità di Roma scrive alla comunità di Corinto (il nome dell'autore, Clemente, è trasmesso dalla tradizione del II secolo, ma la lettera ha un mittente collettivo, «la chiesa di Dio che vive da straniera a Roma»), per esortarla a ristabilire la pace interna, il che, in pratica, significa soprattutto ricollocare al loro posto i presbiteri destituiti. In quel periodo, fine I secolo, infatti sia la chiesa di Corinto che quella di Roma sono guidate da un collegio di presbiteri-anziani, la cui funzione non si è ancora pienamente tecnicizzata ${ }^{17}$. La lettera, da quando è stata «riscoperta» in età moderna, è al centro di una annosa controversia storiografica che riguarda sia in suoi contenuti ecclesiali sia la sua valutazione letteraria. Ma, per quanto riguarda la lingua greca di IClem, siamo su un terreno sicuro perché l'uso stesso dei Settanta la inserisce nell'ambito del giudaismo ellenistico: si tratta cioè della lingua riscontrabile nella produzione letteraria della diaspora. I Settanta prevalgono sul Nuovo Testamento, in quanto in comune con questo ci sono soprattutto i vocaboli paolini, mentre sono di più le parole in comune con i Settanta che non si ritrovano nel Nuovo

precisamente l'analisi di G.A. Kennedy e altri che usano le categorie della retorica classica per descrivere la retorica del NT (p. 2), proponendone invece un'interpretazione socio-retorica. Per parte mia penso che le tematiche individuano indubbiamente un proprium, ma le categorie retoriche sono inevitabilmente contaminate dalla retorica classica, pena, come ho detto, l'impossibilità di farsi ascoltare.

${ }^{17}$ Mi permetto di rinviare alla mia introduzione alla Prima lettera di Clemente in E. Prinzivalli, M. Simonetti (eds.), Seguendo Gesù. Testi cristiani delle origini. I, Milano, 2010, pp. 79-106. 
Testamento. Tuttavia, siccome le parole in comune solo con i Settanta ma non con altri testi del giudaismo ellenistico, sono pochissime, si può concludere che il vocabolario di IClem, nonostante l'ottima conoscenza dei Settanta, non è marcato da espressioni specifiche di questo testo ${ }^{18}$.

Se passiamo alla struttura compositiva della lettera ed esaminiamo in successione le opinioni degli studiosi vediamo che in centocinquant'anni le posizioni iniziali si sono ribaltate. Per oltre un secolo, infatti, si era sottolineata la disorganicità dell'impianto, la frequenza degli excursus, al punto che si vedeva in IClem un affastellamento di temi i più vari, uniti solo in maniera estrinseca dallo scopo dichiarato e si attribuiva tale disorganicità alla derivazione omiletica della lettera ${ }^{19}$. Dopo una fase di furore sezionatorio della lettera (piuttosto sterile in quanto, se la lettera derivava da attività omiletica, di per sé orale, non necessariamente implicava che ci fossero testi scritti presistenti a disposizione dell'autore) da metà degli anni Settanta del secolo passato cominciano $\mathrm{i}$ tentativi di trovare una struttura a un testo che appare unificato solo dal suo scopo. Quello più audace è di Brunner, ingegnoso quanto macchinoso ${ }^{20}$, che individuava una struttura a dittico perfettamente simmetrica e ricca di precisi rimandi fra le due parti. Indissolubilmente intrecciato con il problema della struttura è quello del genere letterario. La soluzione sembra ovvia: IClem è una lettera, reale e non fittizia, e quindi ha tutti i caratteri del genere epistolare: praescriptum, corpo, e postscriptum. Ma il corpo, molto più lungo anche di quello della Lettera ai Romani, la più lunga di Paolo, l'apparenta piuttosto a un trattato. L'autore stesso qualifica in modi diversi lo scritto. Già Wrede pensò che si dovesse prestare attenzione alla definizione data dall'autore della lettera come



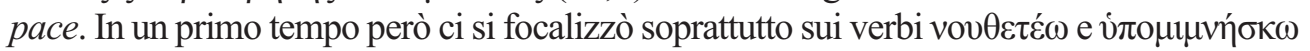

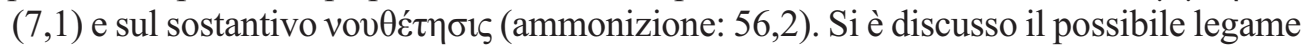

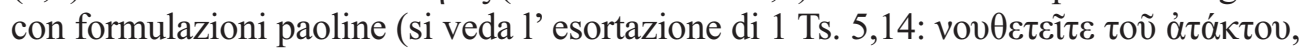
correggete i disordinati!) che hanno fatto pensare a un esercizio di correzione, del tipo di quello che si riscontra in 1 Cor 5,1-5, più o meno formalizzato in senso autoritativo ${ }^{22}$.

A partire dal concetto di ammonizione, IClem è stata considerata come la prosecuzione e l'adattamento a nuove esigenze dell'omelia predicata nelle sinagoghe della diaspora, che dalla cultura ellenistica avrebbe mutuato i caratteri della diatriba cinicostoica. Abbiamo visto sopra la teoria che brani omiletici siano stati rifusi nella lettera. In

${ }^{18}$ Cfr. H.E. Lona, Der Erste Clemensbrief, Göttingen, 1998, p. 35.

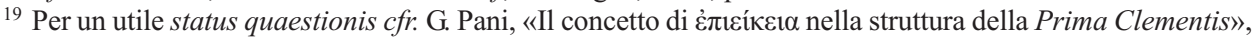
M. F. Wiles, E.J. Yarnold, P.M. Parvis (eds.), Studia Patristica 11, Leuven, 2006, pp. 475-481.

${ }^{20}$ G. Brunner, Die theologische Mitte des ersten Klemensbriefes. Ein Beitrag zur Hermeneutik frühcristlischer Texte, Frankfurt, a. M., 1972.

${ }^{21}$ W. Wrede, Untersuchungen zum Ersten Klemensbriefe, Göttingen, 1891, pp. 1-57. Per una valorizzazione del termine ع̌v $v \varepsilon v \xi 1 \varsigma$ vedi anche A. Lindemann, Die Clemensbriefe, Tübingen, 1992, pp. 13-14; e E. Peretto, Clemente Romano. Lettera ai Corinzi, Bologna, 1999, pp. 54-55.

${ }^{22}$ O. Knoch, Eigenart und Bedeutung der Eschatologie im theologischen Aufriss des ersten Clemensbriefes. Eine auslegunsgeschichtliche Untersuchung, Bonn, 1964, p. 40, insiste molto sul carattere autoritativo dell'ammonizione; nega questo carattere G. Brunner, op. cit., p. 147, n. 20. Riprende questa linea E. Cattaneo, «La Prima Clementis come un caso di correptio fraterna», P. Luisier (ed.), Studi su Clemente Romano. Atti 
questo caso si pensa piuttosto che il corpo della lettera assuma la forma di un'omelia ${ }^{23}$. Per il consolidamento di questa presa di posizione fu determinante l'influsso di un lavoro di Hartwig Thyen, allievo di Rudolf Bultmann, sullo stile dell'omelia giudeo-ellenistica $^{24}$. Thyen rintracciava una serie di figure retoriche, evidenziava analogie con la diatriba $^{25}$, l'equivalente greco di una predica, studiava la parenesi e il modo di citare la Scrittura, nonché le modalità di comunicazione con l'ascoltatore. Tuttavia un problema resta irrisolto: tutti gli esempi vengono da Thyen ricondotti al genere omiletico deduttivamente, in quanto nessun testo a disposizione si presenta formalmente come un'omelia o è tramandato come tale. Lo studioso fa infatti appello ad alcuni testi cristiani (IClem, appunto, la Lettera agli Ebrei, la Lettera di Giacomo, il discorso di Stefano in Atti 7, i capp. 1-6 della Didachè, ecc.) e a opere giudaiche di vario tipo, o a parti di opere giudaiche (il Commento a Genesi di Filone, il IV libro dei Maccabei, il Testamento dei dodici patriarchi). Quindi, per quanto ragionevoli possano essere l'indagine e i suoi presupposti, siamo in presenza di un alto grado di ipoteticità. Thyen aveva omesso di prendere in considerazione qualche altra fonte giudaica ${ }^{26}$, ma di fatto il suo lavoro viene ancora citato perché, stanti le attuali conoscenze, il complesso della documentazione non si è allargato. Lawrence Wills, in un saggio del 1984, prende come esempio-base per studiare la forma del sermone il discorso di Paolo in Atti 13,14-41, chiamato dai capi della sinagoga di Pissidia $\lambda o ́ \gamma o t ~ \pi \alpha \rho \alpha \kappa \lambda \eta ́ \sigma \varepsilon \omega \varsigma$ (parola d'esortazione), che egli definisce «una tipica omelia sinagogale» ${ }^{27}$, e prosegue con la Lettera agli Ebrei, con IClem, altri testi dagli Atti e alcune lettere paoline. Per le fonti giudaiche si limita a pochissimi esempi, fra cui il Testamento dei dodici patriarchi. La sua tesi è che l'omelia cristiana e giudaico-ellenistica presenta uno schema basato sulla successione: esempi, conclusione, esortazione, ripetuti in modo ciclico, in cui l'ultimo elemento, l'esortazione, può essere usato in due direzioni: o per fare da supporto alla conclusione che precede oppure per anticipare i successivi esempi e la successiva conclusione ${ }^{28}$.

degli incontri di Roma, 29 marzo e 22 novembre 2001, Roma 2003, p. 96. La sua ipotesi, presentata in modo accurato, si basa però sul presupposto, anacronistico, che le chiese di Corinto e Roma siano una stessa comunità, nel senso che a Roma sia possibile applicare giuridicamente la correptio, con possibilità di comminare sanzioni.

${ }^{23}$ Così L. Sanders, L'hellénisme de saint Clément de Rome et le paulinisme, Louvain, 1943, pp. 109-130; O. Knoch, op. cit., p. 43.

${ }^{24}$ H. Thyen, Der Stil der Jüdisch-Hellenistischen Homilie, Göttingen, 1955.

${ }^{25}$ H. Thyen, ibid., pp. 40-68 e pp. 47-58. Thyen si basa su uno studio di Bultmann 1910, riguardante l'influsso della diatriba sulla Lettera ai Romani. Il concetto di diatriba, come è assunto da Bultmann e Thyen è stato sottoposto successivamente a critica da S.K. Stowers, The Diatribe and Paul's Letter to the Romans, Ann Harbor, 1981, pp. 175-184, che rivendica a Paolo l'uso della diatriba come tipo di discorso impiegato nella scuola filosofica. Per una bibliografia ragionata sul rapporto fra Paolo e la diatriba $c f r$. R. Burnet, op. cit., pp. 410-411.

${ }^{26}$ Non tiene conto per esempio dell'omelia ps. filoniana su Giona su cui $c f r$. F. Siegert, Drei hellenistischjüdische Predigten, II, Tübingen, 1992, pp. 40-47.

${ }^{27}$ L. Wills, «The Form of the Sermon in Hellenistic Judaism and Early Christianity», Harvard Theological Review 77 (1984), pp. 277-299.

${ }^{28}$ Per far comprendere in che oscurità gli storici si muovono a proposito dell'omelia riporto il parere aggiornato di L. I. Levine, La sinagoga antica.2. L'istituzione, Brescia, 2005 (ed. orig. 2000), pp. 168-169: «C'è chi ha ritenuto che 4 Maccabei sia una serie di sermoni sinagogali, che le domande e risposte di Filone siano nate in un tale ambiente, o che i discorsi degli Atti testimonino modelli di sermoni giudeo-ellenistici e anticipino più recenti modelli omiletici. Nessuna ipotesi del genere, pur suggestiva, risulta pienamente convincente». 
Jaeger aveva offerto i primi spunti per una via diversa, che passa per un maggior apprezzamento generale della cultura greco-romana come elemento formativo del linguaggio e della concettualizzazione del messaggio evangelico. Soffermandosi sul termine $\pi \alpha 1 \delta \varepsilon i ́ \alpha$ (educazione, istruzione) in IClem 56,2, vede nel suo uso la traduzione di un concetto pagano, ammirato dagli ebrei e quindi dai cristiani ${ }^{29}$, nei termini di istruzione derivante da Dio. Egli inoltre sottolinea l'importanza del termine ónóvola (concordia) e ipotizza la collocazione della lettera nel genere deliberativo o symbouleutikon, che ha lo scopo di persuadere a prendere la decisione più vantag$\operatorname{giosa}^{30}$. Avanza con decisione su questa strada van Hunnik ${ }^{3}$, il quale parte dalla duplice constatazione del ruolo centrale giocato nella lettera dal termine ó $\mu$ óvor $\alpha$, talvolta

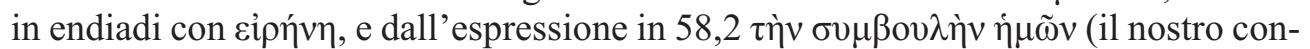
siglio), che sembra riferirsi all'intera lettera, per sviluppare la tesi che IClem appar-

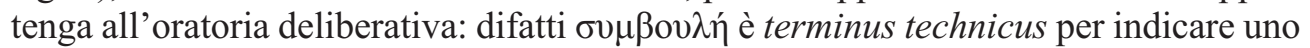
dei tre generi di discorso, secondo Aristotele (Ars Reth. 1,3). Con una ampia serie di esempi tratti da oratori greci (Isocrate, Dione Crisostomo, Elio Aristide) egli mostra la connessione costante della semantica della concordia con quella dell'avviso, consiglio, la $\sigma v \mu \beta 0 v \lambda \eta ́$ appunto, in vista del ristabilimento della pace. La posizione di van Hunnik introduce negli studi una maggiore attenzione verso la retorica antica come elemento strutturante il linguaggio di IClem. Sulla scorta di van Hunnik di recente Odd Magne Bakke ${ }^{32}$ si è assunto l'onere di compiere una lettura complessiva della lettera in funzione della retorica antica, sui cui risultati specifici vale quanto detto sopra a proposito di Paolo: il genere epistolare non sopporta un grado eccessivamente elevato di coerenza strutturale, e non può essere trattato alla stregua di un'orazione. Si è dunque percorsa, nell'ultima fase della critica, anche per Clemente l'esperienza fatta con le lettere di Paolo. Nessuno potrà d'altra parte negare, visto che la lettera è intessuta di citazioni scritturistiche in massima parte perfettamente corrispondenti al testo dei Settanta, che l'autore, qualunque sia la sua origine, abbia avuto anche una accurata istruzione giudaica, e del resto sappiamo che il cristianesimo a Roma si sviluppa con una facies giudaica pronunciata. Difatti tutti gli studiosi impegnati ad approfondire l'impostazione retorica di IClem, compreso il più acceso

\footnotetext{
${ }^{29}$ W. Jaeger, «Early Christianity and the Greek Paideia», C. Breytenbach, L.L. Welborn (eds.), Encounters with Hellenism. Studies in the First Letter of Clement, Leiden-Boston, 2004, pp. 104-114 (lo studio di Jaeger, qui ripubblicato, è del 1961).

${ }^{30}$ W. Jaeger, «Echo eines unbekannten Tragikerfragmentes in Clemens' Brief an die Korinther», Rheinisches Museum für Philologie, 102 (1959), pp. 330-340.

31 W.C. van Unnik, Studies over de zogenaamde Eerste Brief vam Clemens, Mededelingen, 1970, pp. 149204, ripubblicato in inglese in C. Breytenbach, L.L. Walborn (eds.), op. cit., pp. 115-181.

32 O. M. Bakke, «Concord and Peace». A Rhetorical Analysis of the First Letter of Clement with an Emphasis on the Language of Unity and Sedition, Tübingen, 2001. Egli distingue il corpus della lettera («Sub Texts on Grade Two») in exordium (1,1-2,8), narratio (3,1-4), una lunga probatio $(4,1-61,3)$ e peroratio $(62,1-64,1)$. Appoggiandosi poi alla suddivisione proposta all'interno della probatio da alcuni antichi teorici -su tutti Ermogene di Tarso- fra una $\theta \varepsilon \dot{\varepsilon}$ ı/quaestio infinita/quaestio generalis, cioè un approccio astratto e generale al problema, e una

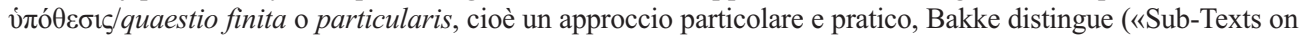
Grade Three») la probatio in due parti, la prima (quaestio infinita) da 4,1 a 39,9 e la seconda (quaestio finita) da 40,1-61,3. L'ulteriore analisi individua le macro-strutture in cui si snoda la composizione.
} 
fautore della sua coerenza con il genere deliberativo, cioè Bakke, non se la sentono di negare l'apporto della componente omiletica, per quanto sfuggente nei dettagli esso sia: è un fatto che l'omelia sinagogale esisteva e che anche i fedeli in Cristo, già nel I e II secolo, ascoltavano prediche, come risulta ad esempio da Giustino ${ }^{33}$. A questo proposito, se Wills aveva suggerito ${ }^{34}$ un confronto con la retorica greca per concludere che lo schema da lui individuato non combacia con le partizioni del discorso oratorio, C. Clifton Back II, rispondendogli ${ }^{35}$, lo critica proprio su questo punto: a suo avviso gli standard della retorica classica, assimilati da giudei e cristiani, servono da base per capire le forme della predicazione. Siamo di fronte a un circolo vizioso? Forse. Questo dovrebbe indurci a prendere atto del punto centrale della questione: non è possibile prescindere, per chi conosce bene la lingua greca, come è il caso del nostro autore, dalle modalità retoriche elaborate dai greci e largamente assimilate nel giudaismo della diaspora. L'autore della lettera ha una predilezione per i libri sapienziali della Scrittura. Ebbene, già gli antichi si erano resi conto dell'affi-

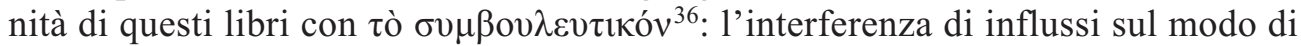
argomentare di IClem dovrebbe a questo punto essere sufficientemente chiara a chi abbia avuto la pazienza di seguirmi.

Recenti studi ${ }^{37}$ hanno puntualizzato che la quasi totalità della terminologia qualificata in passato come filosofica si ritrova negli scrittori del giudaismo ellenistico, in particolare in quell'autore filosofico per eccellenza che è Filone. La diffusione a Roma delle sue opere è questione controversa. In ogni caso il lungo soggiorno a Roma (39/40) di Filone per l'ambasceria a Caligola è un dato certo, e ciò implica che una certa circolazione del suo pensiero sia avvenuta. A Roma soggiornò anche Flavio Giuseppe. A proposito di IClem, Runia conclude che uno sfondo giudaico-ellenistico, a prescindere dal problema della conoscenza dei singoli autori, è incontrovertibile ${ }^{38}$. Sopra avevamo già visto che la lingua di IClem si caratterizza come appartenente all'alveo della letteratura giudaico ellenistica, declinata secondo la nuova fede sorta in seno al giudaismo. Egli doveva necessariamente rientrare in una fitta rete di rapporti sociali e di comunicazione intellettuale, in forza del suo ruolo di portavoce di una comunità, e, prima ancora, in forza della sua estrazione, ignota, ma sicuramente collegata a ceti elevati, in quanto gli aveva reso possibile acquisire il grado di cultura documentabile dall'opera, con esso buone relazioni e di conseguenza la possibilità di accesso al patrimonio culturale giudaico come a quello pagano.

33 Giustino, 1Apol. 67,4.

${ }^{34}$ L. Wills, op. cit., pp. 296ss.

${ }^{35}$ C. Clifton Black II, «The Rhetorical Form of the Hellenistic Jewish and Early Christian Sermon: A Response to Laurence Wills», Harvard Theological Review 81 (1988), pp. 1-18.

${ }^{36} \mathrm{Cfr}$. G. Dorival, «Has the Category of 'Deuterocanonical Books' a Jewish Origin?, G.G. Xeravits, J. Xsengellér (eds.), The Books of the Maccabees: History, Theology, Ideology. Papers of the Second International Conference on the Deuterocanonical Books, Pápa, Hungary, 9-11 June 2005, Leiden, 2007, p. 4, n. 13. 5-35.

${ }^{37}$ Cfr. soprattutto J. P. Martín, «Prima Clementis: ¿Estoicismo o Filonismo?», Salmaticensis 41 (1994), pp.

${ }^{38}$ D.T. Runia, Filone di Alessandria nella prima letteratura cristiana. Uno studio di insieme, Milano, 1999, p. 98 . 
La collocazione di IClem nel quadro del giudaismo ellenistico conduce a ridimensionare drasticamente la visione della sua dipendenza dalla filosofia, secondo la proposta unilaterale di Sanders, che provocò la risposta altrettanto unilaterale di Beyschlag, sull'esclusiva dipendenza di IClem da tradizioni giudaiche e giudaico-cristiane ${ }^{39}$. Prevale attualmente una visione inclusiva della cultura del nostro autore. Il suo livello di istruzione, ripeto, comprende di necessità la conoscenza di una parte almeno del patrimonio letterario ellenistico, mentre sulle conoscenze filosofiche bisogna essere prudenti perché dalla lettera emergono echi piuttosto generici, senza precisi elementi di dottrina. Le sue idee sulla comunità cristiana si giovano della retorica sulla concordia, di cui troviamo numerosi esempi in oratori greci di poco posteriori. Egli si spinge fino a riconoscere l'esemplarità di uomini e donne pagane (IClem 55), un fatto che colpì anche Origene ${ }^{40}$, ma, a differenza di come agisce quando tratta di personaggi della storia di Israele, non ne ricorda i nomi. Analogamente, non riusciamo a distinguere una dipendenza sicura da nessuna opera pagana. La sua capacità di interiorizzare e riplasmare il patrimonio giudaico si esercita ugualmente e anche di più nei confronti di quello greco.

Un aspetto interessante dell'assimilazione dell'ellenismo nel nostro autore è, come dicevo, il suo uso del linguaggio della concordia civica. Un riscontro puntuale ne dimostra l'intenzionalità. Oltre alla parola chiave homonoia fa la sua comparsa in IClem il termine $\sigma \tau \alpha \dot{\sigma} \sigma \varsigma \varsigma$, di centrale importanza: ricorre nove volte, anche in endiadi, mentre il corrispondente verbo $\sigma \tau \alpha \sigma i \alpha ́ \zeta \omega$ sette volte ${ }^{41}$. Viene abitualmente usato in riferimento alle guerre civili, o a discordie interne, al contrario di $\pi$ ó $\lambda \varepsilon \mu \rho \varsigma$, che indica la guerra fra

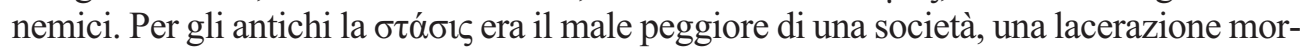
tale del suo corpo. Evidentemente IClem lo giudica adeguato proprio in forza della sua risonanza così negativa. La sua posizione è tanto più significativa in quanto Paolo, nel riferirsi ai dissensi dei Corinzi, non lo adoperava ${ }^{42}$. La scelta di IClem va compresa nel quadro di una consapevole tendenza a definire i rapporti interni alle comunità dei fedeli in Cristo con la terminologia consueta nel mondo ellenistico per indicare le dinamiche sociali e politiche. Le chiese di Dio secondo IClem sono entità differenziate rispetto all'ambiente circostante (infatti sono come stranieri residenti) ma hanno dinamiche interne analoghe a quelle di ogni corpo sociale, sia pur risostanziate di significato. Negli altri cosiddetti Padri Apostolici il termine non è usato. E' invece interessante constatarne

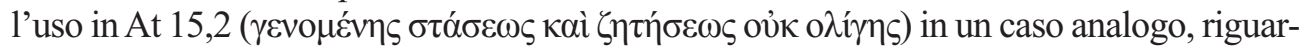
dante cioè discussioni interne, precisamente fra «alcuni dalla Giudea» che volevano circoncidere i fratelli pagani e Paolo e Barnaba. In varie espressioni di IClem si fondono echi della religione politica ellenistica e della mentalità giudaica. Possiamo confron-

\footnotetext{
${ }^{39}$ L. Sanders, op. cit., Tübingen, 1966.

${ }^{40}$ Cfr. Origene, Commento a Giovanni I 29, 279; ibid., VI 54, 279; XXVIII 19, 162; Contro Celso I, 31 (nelle ultime due occorrenze non nomina IClem, ma deriva da lui la notizia).

${ }^{41}$ Cfr. l'indice di A. Jaubert, Clément de Rome. Épître aux Corinthiens (reimpression de la première édition revue et corrigée) (Sources Chrétiennes, 167), Paris, 2000, p. 268.

${ }^{42}$ Cfr. M .M. Mitchell, Paul and the Rhetoric of Reconciliation. An Exegetical Investigation of the Language and Composition of ICorinthians, Tübingen, 1991, pp. 76-80 e n. 66.
} 
tare tale espressioni di IClem con alcune molto vicine di un autore fondamentale per la retorica civica, anche se cronologicamente posteriore, Elio Aristide, Oratio 24.48: «la

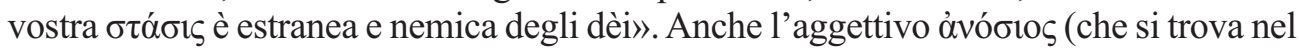
Nuovo Testamento solo in 1 Tim 1,9 e 2 Tim 3,2) può rientrare nel quadro ${ }^{43}$. Si potrebbe dire che un vocabolario del genere opera una costruzione contro-culturale dell'ordine nella chiesa quale specchio capovolto dell'ordine e degli ideali imperiali ${ }^{44}$. In IClem 1,2

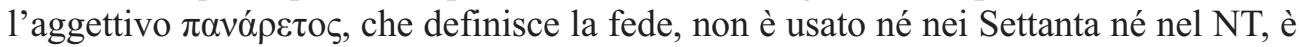
usato una sola volta in Filone e mai in Flavio Giuseppe, al contrario di $\beta \varepsilon \beta \alpha i \alpha$, consueto nel giudaismo ellenistico ${ }^{45}$. A proposito di questa doppia aggettivazione che accompa-

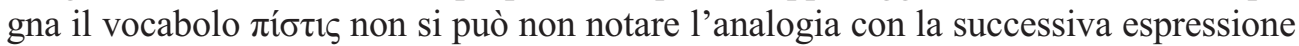

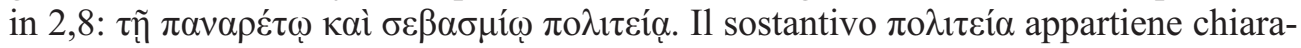
mente al linguaggio civico e la doppia aggettivazione serve a inserire in esso una sfumatura anche religiosa. Qualcosa di analogo, ma in direzione opposta, potrebbe darsi quindi anche nel presente caso: il sostantivo $\pi$ í $\tau$ tı è fondamentale nella letteratura cristiana delle origini, neotestamentaria e non, ma lo è anche nel mondo greco, nel senso di fiducia, rispetto dei patti che è alla base della concordia civica ${ }^{46}$. Siamo quindi di fronte all'inglobamento di un significato civico in una terminologia prevalentemente religiosa.

Lo stesso fenomeno su cui mi sono soffermata in Clemente, si riscontra in Ignazio di Antiochia, di circa venti anni posteriore e di altro ambiente (Antiochia di Siria), ma ugualmente coinvolto nella retorica della seconda sofistica e nel linguaggio civico. Lo studioso che più ha analizzato sotto questo profilo le sue sette lettere autentiche è Allen Brent e la sua disamina, nonostante talune forzature, può essere recepita come valida $^{47}$.

Possiamo fare un esempio della sua prosa, nella quale si potrà apprezzare la riformulazione di immagini tradizionali e l'accostamento fra l'immagine del coro nell'armonia musicale e il concetto di concordia interna. Ebbene lo stesso accostamento ritroviamo, all'incirca negli stessi anni, in Dione di Prusa, orat. 36. Ovviamente non sto cercando di stabilire dipendenze, ma di segnalare le affinità di uno stesso universo mentale, pur nella novità dei contenuti cristiani.

Così dunque dice Ignazio nella lettera agli efesini, cap. 4:

4.1. Perciò è conveniente che siate d' accordo col pensiero del vostro vescovo, come effettivamente fate. Infatti il vostro presbiterio, di chiara fama e degno di Dio, è in perfetta sintonia col vescovo, come le corde con la cetra ${ }^{48}$. Perciò grazie alla

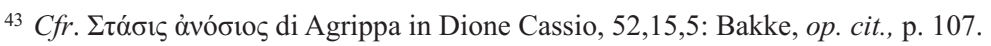

${ }^{4}$ A. Brent, The Imperial Cult and the Development of Church Order. Concept and Images of Authority in Paganism and Early Christianity before the Age of Cyprian, Leiden, 1999, pp. 140-163; Id., Ignatius of Antioch, pp. 245-255.

${ }^{45}$ H. E. Lona, op. cit., p. 121.

46 Bakke, op. cit., pp. 108-114.

${ }^{47}$ Vedi soprattutto il suo Ignatius of Antioch and the Second Sophistic. A Study of an Early Christian Transformation of Pagan Culture, Tübingen, 2006. Cfr. anche il successivo A Political History of Early Christianity, London- New York, 2009, pp. 195-208.
} 
vostra concordia e all' armonia del vostro amore, viene esaltato col canto Gesù Cristo. 2. Tutti voi, uno per uno, possiate diventare un coro, affinché in armoniosa concordia, prendendo da Dio l' accordo, cantiate tutti all' unisono rivolti al Padre per tramite di Gesù Cristo, acciocché egli vi presti ascolto e riconosca, grazie alle vostre buone opere, che voi siete membra del suo figlio. E' bene perciò che voi siate irreprensibilmente uniti, per essere sempre partecipi di Dio.

Lo stile di Ignazio, lo notava già Norden ${ }^{49}$, fa violenza spesso alla grammatica. D'altra parte le caratteristiche asiane del suo stile, dal procedere per brevi frasi collegate da anafore, omoteleuti e da grande varietà di figure retoriche rendono indubbia la pretesa letteraria di Ignazio. Se Norden spiegava le sgrammaticature con l'urgenza e l'ardore di un carattere passionale, di recente Manlio Simonetti ipotizza che possa trattarsi di un connubio non inusuale fra carenze grammaticali di base e ambizioni retoriche ${ }^{50}$. Ma anche in quest'ultima ipotesi l'evidenza retorica del testo risulta confermata. Il II secolo vedrà il confronto dei cristiani con il mondo pagano ma anche con quello giudaico, dal quale si vuole marcare una separazione. Tanto più sarà necessaria l'arte retorica $\mathrm{e}$ un'impostazione più o meno embrionalmente filosofica per gli apologeti. Ma, come abbiamo visto, l'efficacia nella comunicazione scritta, la capacità di costruire il discorso, manifestatasi subito nel cristianesimo serve innanzitutto alla regolazione dei rapporti interni alla comunità, «alla conservazione e modifica dei rapporti di potere nell'ambito delle comunità cristiane». Scrivere per governare, dunque ${ }^{51}$. La parola, o il logos, nel caso del cristianesimo fondato sulla parola/Logos per eccellenza, dunque è sovrana, come era sovrana nel mondo ellenico. I cristiani condivisero tutto ciò e lo usarono ad intra e ad extra in un mondo che era senza pietà per chi non sapeva usare le parole: come probabilmente capitò alle due malcapitate ma coraggiose schiave ministrae ${ }^{52}$ interrogate sotto tortura dall'intellettuale Plinio il Giovane.

\footnotetext{
${ }^{48}$ Per un parallelo di metafora musicale applicata all'armonia nella città $c f r$. Dione Crisostomo, Orationes 36, 21: cfr. L. Brent, op. cit., p. 238 ss.

${ }^{49}$ E. Norden, La prosa d'arte antica dal VI secolo a.C. all'età della Rinascenza, B. Heinemann Campana (reed. it.), con una nota di aggiornamento di G. Calboli e una premessa di S. Mariotti, I-II, Roma, 1986 (ed. orig. 1915) ${ }^{3}$.

${ }^{50}$ M. Simonetti, in E. Prinzivalli, M. Simonetti (eds.), op. cit., p. 301.

${ }^{51}$ Come recita il titolo di un bel convegno tenutosi a Brescia nel 2005, pubblicato l'anno dopo nella Rivista Storia del cristianesimo 3.1 (2006), a cura di E. Norelli.

52 Plinio il Giovane, ep. X, 96.
} 